\title{
Conforto térmico de bovinos leiteiros confinados em clima subtropical e mediterrâneo pela análise de parâmetros fisiológicos utilizando a teoria dos conjuntos fuzzy
}

\author{
Thermal comfort on Subtropical and Mediterranean climate analyzing some physiological data \\ through fuzzy theory
}

\author{
Maurício Perissinotto ${ }^{\mathrm{I}}$ Daniella Jorge Moura ${ }^{\mathrm{II}}$ Vasco Fitas Cruz ${ }^{\mathrm{III}}$ Silvia Regina Lucas de Souza \\ Karla Andréa Oliveira de Lima ${ }^{\text {II }}$ Angélica Signor Mendes ${ }^{\text {IV }}$
}

\section{RESUMO}

Os objetivos deste estudo foram modelar e avaliar, pelo uso da lógica fuzzy, a sensação de conforto térmico de animais confinados em função das variáveis fisiológicas temperatura retal (TR) e frequência respiratória (FR), determinando os intervalos críticos dessas variáveis. O banco de dados foi formado em dois ambientes distintos: clima subtropical (Município de São Pedro, Brasil) e clima mediterrâneo (Município de Évora, Portugal). Para a formação do banco de dados fisiológicos, foram obtidos dados de TR e FR de vacas holandesas. Para a análise física do ambiente, foram utilizados dados de estações meteorológicas com leituras de temperatura e umidade relativa do ar realizadas a cada 30 min, ao longo de 24 horas. No processo inicial de análise dos dados, foi utilizada a técnica de Mineração de Dados com o objetivo de formar uma árvore de decisão para a indução de regras. Para isso, foi utilizado o programa computacional WEKA ${ }^{\circledR}$. Os resultados obtidos foram posteriormente utilizados na aplicação da lógica fuzzy, em que foi utilizado o software Fuzzy Logic Toolbox do MATLAB ${ }^{\circledR}$ 6.1, seguindo as recomendações de AMENDOLA et al. (2005b). A utilização dessa ferramenta permitiu estabelecer alguns parâmetros ideais de conforto aos bovinos leiteiros da raça Holandesa em lactação manejados em condição de confinamento total.

Palavras-chave: produção animal, estresse térmico, lógica fuzzy, bovinos leiteiros.

\section{ABSTRACT}

The objective of this study was to model and evaluate, through fuzzy logic, the level of thermal comfort experienced by housed animals as a function of their physiologic variables of rectal temperature (RT) and breath rate (BR), and setting their critical thresholds. The database was setup using two distinct environments: Subtropical climate (São Pedro area, Brazil) and mediterranean climate (Évora area, Portugal). Holstein cows temperature and breath rates were obtained in order to build a physiologic parameters database. meteorological data of environment temperature and air relative humidity were obtained for physical analysis during a 24 hours interval every 30 minutes. Data minning techniques were used for the initial data analysis aiming to build a decision making three and the further construction of rule database. For that, the computational program WEKA ${ }^{\circledR}$ was used. The results obtained were applied for the fuzzy logic application, using the Fuzzy Logic Toolbox do MATLAB ${ }^{\circledR} 6.1$ software, according to AMENDOLA et al. (2005b). The use of this tool allowed the establishment of thermal comfort parameters for total confined Holsteins cows.

Key words: animal production, thermal stress, fuzzy logic, milking cattle.

\section{INTRODUÇÃo}

Devido à constante necessidade de produção de alimentos em grande escala, o confinamento de animais surgiu como uma alternativa para o aumento da produtividade, por meio do controle das condições ambientais na área de alojamento. Dessa forma, novos problemas apareceram com os sistemas de confinamento , mas também novas oportunidades para manejar rebanhos leiteiros com melhor conforto, permitindo melhores níveis de produção sem comprometer aspectos reprodutivos e de saúde geral

ICentro de Tecnologia, Universidade Estadual de Campinas (Unicamp), Campinas, SP, Brasil.

IIFaculdade de Engenharia Agrícola, Unicamp, Cidade Universitária Zeferino Vaz, Barão Geraldo, CP 6011, 13083970, Campinas, SP, Brasil. E-mail: daniella.moura@agr.unicamp.br. *Autor para correspondência.

IIIDepartamento de Engenharia Rural, Universidade de Évora, Portugal.

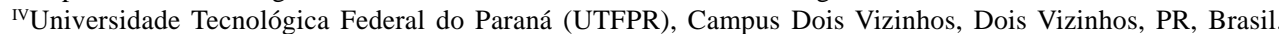


dos animais. Nesse contexto, fatores ambientais externos podem interferir no microclima gerado no interior das instalações, causando com isso impactos sobre a produção, com consequentes prejuízos econômicos à exploração. Uma forma de avaliar as respostas dos animais ao ambiente térmico é por meio da observação de alguns parâmetros fisiológicos, como a temperatura retal (TR) e a freqüência respiratória (FR).

A medida da temperatura retal orienta a determinação do equilíbrio entre o ganho e a perda de calor do corpo, sendo essa medida usada frequentemente como índice de adaptabilidade (MOTA, 1997). Para HAHN et al. (1997), o aumento da FR, quando considerado por curto período, é um mecanismo eficiente de perda de calor. Porém, quando os valores ultrapassam 120 movimentos respiratórios por minuto $\left(\right.$ mov $\left.\min ^{-1}\right)$, o animal está sofrendo com a carga excessiva de calor e, acima de $160 \mathrm{mov} \mathrm{min}^{-1}$, as medidas de emergência devem ser tomadas a fim de amenizar o estresse.

As variações da TR e da FR podem ser influenciadas, tanto por fatores intrínsecos (idade, raça, estado fisiológico), quanto por fatores extrínsecos (hora do dia, ingestão de alimentos e de água, temperatura ambiente, velocidade do vento, estação do ano).

Em ambientes agrícolas controlados, tais como as instalações de produção animal, torna-se necessário o uso de técnicas de controle das variáveis ambientais. A Mineração de Dados e a lógica fuzzy, dentre outras ferramentas direcionadas para a tomada de decisão e para ações mais precisas, têm contribuído para o avanço e a velocidade das pesquisas em produção animal. Diversos trabalhos utilizaram a lógica fuzzy como ferramenta de suporte à decisão na agropecuária. Como exemplo, na bovinocultura, SANTOS \& NÄÄS (2006) utilizaram a lógica fuzzy para simular a taxa de detecção de cio de vacas leiteiras confinadas e expostas a diferentes condições de temperatura (T) e umidade relativa do ar (UR), e BRUNASSI et al. (2006) conseguiram encontrar 100\% de eficiência na detecção de estro em vacas leiteiras com um sistema baseado em lógica fuzzy. A aplicação da teoria fuzzy vem sendo utilizada também nas áreas de ambiência e produção animal (GATES et al., 1999; AMENDOLA et al., 2005a; OLIVEIRA et al., 2005), comprovando a eficácia do uso dessa ferramenta nesses estudos.

O objetivo deste estudo foi estimar o conforto térmico de bovinos leiteiros confinados a partir de duas variáveis fisiológicas: temperatura retal e frequência respiratória, utilizando a lógica fuzzy.

\section{MATERIAL E MÉTODOS}

Neste experimento, foram utilizadas vacas leiteiras holandesas multíparas e em lactação. Os animais foram divididos em lotes de acordo com a produção de leite e/ou período de lactação e confinados em abrigo do tipo freestall.

O ambiente térmico exerce forte influência sobre o desempenho animal, uma vez que afeta os mecanismos de transferência de calor e, assim, a regulação do balanço térmico entre o animal e o meio. $\mathrm{O}$ animal dentro de um ambiente térmico considerado adequado produzirá de acordo com o seu potencial genético; porém, existem vários relatos sobre esses limites. Tendo em vista que por meio de bancos de dados fisiológicos e de estações meteorológicas há a possibilidade de modelagem e avaliação do impacto do estresse térmico e sabendo-se que os limites do ambiente térmico podem sofrer variações em função da região e dos animais utilizados na propriedade, a obtenção dos dados fisiológicos e a formação do banco de dados foram desenvolvidas em dois ambientes distintos: clima subtropical (ambiente 1) e clima mediterrâneo (ambiente 2).

- Ambiente 1 (Clima Subtropical): O experimento foi realizado no Município de São Pedro, Brasil. O Município de São Pedro encontra-se na latitude $22^{\circ} 32^{\prime}$ 55" Sul e longitude $47^{\circ}$ 54' 50 " Oeste, a $580 \mathrm{~m}$ de altitude. O clima subtropical é característico das áreas geográficas a Sul do Trópico de Capricórnio e a Norte do Trópico de Câncer, com temperaturas médias anuais nunca superiores a $20^{\circ} \mathrm{C}$ e temperatura mínima do mês mais frio nunca menor que $0^{\circ} \mathrm{C}$. O clima é quente e úmido com estação chuvosa no verão e seca no inverno. A instalação utilizada possui $80 \mathrm{~m}$ de comprimento, 28m de largura, 9m de altura na parte central, 3,5m nas laterais, orientação Leste-Oeste, telhado coberto com telha de barro e piso de concreto. Além disso, possui dois lotes divididos por um corredor central de 2,90m de largura e capacidade de alojamento de 200 animais adultos. Foram utilizadas 15 vacas, e a coleta dos dados fisiológicos foi realizada durante nove dias não-consecutivos do mês de novembro de 2003 às 9h, 11h, 13h, 15h e 17h.

- Ambiente 2 (Clima Mediterrâneo): O experimento foi realizado no Município de Évora, Portugal. A cidade de Évora encontra-se na latitude $38^{\circ}$ 36' e longitude $7^{\circ} 54$ ', a 30m de altitude e está situada na região Sul de Portugal (região do Alentejo). As regiões de clima mediterrâneo são diferentes das regiões de clima subtropical, combinando verões quentes e secos com invernos frios e pouco chuvosos. Como o próprio nome indica, esse tipo de bioma localiza-se na zona do 
mar Mediterrâneo. Ocorre precipitação, sobretudo, durante dois a quatro meses, no inverno, sendo rara no resto do ano. O clima é quente e seco no verão e com estação chuvosa no inverno. A temperatura máxima média anual é de $31,7^{\circ} \mathrm{C}$, e a temperatura mínima média é de $2,5^{\circ} \mathrm{C}$. A instalação utilizada possui $60 \mathrm{~m}$ de comprimento, $20 \mathrm{~m}$ de largura, $9 \mathrm{~m}$ de altura na parte central, 3,5m nas laterais, orientação Leste-Oeste, telhado coberto com telha metálica e piso de concreto. Possui dois lotes divididos por um corredor central de 2,90m de largura e capacidade para alojar 100 animais adultos. Foram utilizados seis animais, e a coleta dos dados fisiológicos foi realizada durante 20 dias nãoconsecutivos nos meses de agosto e setembro de 2005, às $10 \mathrm{~h}, 12 \mathrm{~h}, 14 \mathrm{~h}, 16 \mathrm{~h}$ e $18 \mathrm{~h}$.

Para a análise física do ambiente interior da instalação, em São Pedro, foram utilizados sistemas de aquisição de dados HOBO®. Em Évora há estações meteorológicas da Campbell Scientific ${ }^{\circledR}$, que foram fixadass no centro geométrico da instalação e registraram os valores da temperatura de bulbo seco e da umidade relativa do ar a cada $30 \mathrm{~min}$ ao longo das 24h durante o período de coleta de dados, possibilitando o cálculo do Índice de Temperatura e Umidade (ITU), de acordo com a equação desenvolvida originalmente por THOM (1959): ITU = Tbs + 0,36 Tpo +41 , em que Tbs é a temperatura de bulbo seco $\left({ }^{\circ} \mathrm{C}\right)$, e Tpo é a temperatura do ponto de orvalho $\left({ }^{\circ} \mathrm{C}\right)$.

Os dados fisiológicos coletados foram a temperatura retal (TR) e a freqüência respiratória (FR). Para ambos os ambientes de estudo, a TR foi obtida por um termômetro clínico digital inserido no reto, já a FR, pela contagem dos movimentos da região do flanco, durante 15s. Portanto, foram obtidos para as análises 675 dados de TR e FR, para o ambiente 1, e 600 dados, para o ambiente 2 .

No processo inicial de análise dos dados, foi utilizada a técnica de Mineração de Dados com o objetivo de formar uma árvore de decisão para a indução de regras. Para isso, foi utilizado o programa computacional Waikato Environment for Knowledge Analysis (WEKA ${ }^{\circledR}$ ), versão 3-4, que executou tarefa de classificação, utilizando o algoritmo J48 em validação cruzada para a construção da árvore de decisão. O processo de Mineração de Dados foi dividido em fases, com um conjunto de ações definidas para cada uma delas, desencadeadas de forma cíclica, de acordo com a metodologia CRISP-DM (CHAPMAN et al., 2000). Como resultado final, alcançou-se um conjunto ordenado de regras (cada caminho possível da árvore correspondeu a uma regra do tipo "SE < condicional> ENTÃO < consequente>”).
Na segunda etapa de análise dos dados, a base de regras, baseada no conhecimento adquirido com a Mineração de Dados, foi utilizada para a modelagem do sistema especialista, baseado em lógica fuzzy. Para isso, foi utilizado o software MATLAB ${ }^{\circledR}$ 6.1, no módulo Fuzzy Logic Toolbox. Para a construção da estrutura básica do sistema baseado em regras fuzzy, foram seguidas as recomendações de AMENDOLA et al. (2005b), os quais sugerem a inclusão de quatro componentes principais: um fuzzificador, uma base de conhecimento, um método de inferência e um defuzzificador.

As informações obtidas na etapa de Mineração de Dados foram reorganizadas e classificadas em termos linguísticos. As variáveis de entrada do modelo, ou seja, variáveis independentes foram a TR e a FR e a variável de saída, ou seja, a variável dependente foi a sensação de conforto térmico. Para a variável TR $\left({ }^{\circ} \mathrm{C}\right)$, considerou-se o domínio no intervalo [37; 40], representando as faixas: $<38,8^{\circ} \mathrm{C}$ (conforto alto); $38,8-39,2^{\circ} \mathrm{C}$ (conforto médio); e $>39,2^{\circ} \mathrm{C}$ (conforto baixo). Para a variável FR (mov $\min ^{-1}$ ), considerou-se o domínio do intervalo [28; 108], representando as faixas: $<56$ (conforto alto); 56-64 (conforto médio); e >64 (conforto baixo).

Na construção dos conjuntos fuzzy, foi utilizado um modelo com quatro caracterizações linguísticas em que foram consideradas classificações relativas ao estado de conforto térmico proporcionado pelo sistema de confinamento em: ruim, regular, bom e muito bom, de acordo com as faixas $0,0-0,25 ; 0,25-0,5$; 0,5-0,75; e 0,75-1, respectivamente. Na tabela 1 , é apresentada a classificação do estado de conforto térmico, com seus respectivos pesos, como função da TR $\left({ }^{\circ} \mathrm{C}\right)$ e da FR $\left(\operatorname{mov} \mathrm{min}^{-1}\right)$.

O método de inferência utilizado foi o Método de Mamdani (MANDANI, 1976), e os resultados da defuzzificação foram calculados com a aplicação do método do centro de gravidade, também utilizado por vários autores em pesquisas de mesma natureza, a saber: AMENDOLA et al. 2004; WATANABE et al. 2005; ALVES, 2006; AMENDOLA et al., 2006; BRUNASSI et al. 2006; CAMPOS e AMENDOLA, 2006; CHIARINI et al. 2006; OWADAe NÄÄS, 2006; PANDORFI et al. 2006; SANTOS e NÄÄS, 2006.

\section{RESULTADOS E DISCUSSÃO}

Os dados fisiológicos obtidos em clima subtropical apresentaram uma amplitude de $72 \mathrm{mov} \mathrm{min}^{-1}$ e 4,0 $0^{\circ} \mathrm{C}$ para a freqüência respiratória e temperatura retal, respectivamente, e a FR variou do mínimo de 32mov 
Tabela 1 - Classificação do estado de conforto térmico, com seus respectivos pesos, como função da TR ( $\left.{ }^{\circ} \mathrm{C}\right)$ e da FR (mov min ${ }^{-1}$ ).

\begin{tabular}{llcl}
\hline & & & \\
Temperatura retal $\left({ }^{\circ} \mathrm{C}\right)$ & Conforto alto $(\leq 56)$ & Conforto médio (56-64) & Conforto baixo $(>64)$ \\
\hline Conforto alto $(\leq 38,8)$ & Muito bom $(1,0)$ & Bom $(1,0)$ & Regular $(0,5)$ \\
Conforto médio $(38,8-39,2)$ & Bom $(1,0)$ & Regular $(1,0)$ & Ruim $(1,0)$ \\
Conforto baixo $(>39,2)$ & Regular $(0,5)$ & Ruim $(1,0)$ & Ruim $(1,0)$ \\
\hline
\end{tabular}

$\min ^{-1}$ ao máximo de $104 \mathrm{mov}_{\mathrm{min}^{-1}}$, com uma média de $58 \mathrm{mov} \min ^{-1}$. Já a TR variou do mínimo de $36^{\circ} \mathrm{C}$ ao máximo de $40,0^{\circ} \mathrm{C}$, com média de $38,4^{\circ} \mathrm{C}$. Da mesma forma, as variáveis climáticas e o índice de conforto térmico analisados apresentaram uma alta amplitude, sendo constatados os valores de $17^{\circ} \mathrm{C}$ (mínimo de $21,2^{\circ} \mathrm{C}$ e máximo de $38,2^{\circ} \mathrm{C}$ ), 53,6\% (mínimo de $21,2 \%$ e máximo de 74,8\%) e 17,4 (mínimo de 68,4 e máximo de 85,8) para a Temperatura de bulbo seco $\left({ }^{\circ} \mathrm{C}\right)$ (Tbs), Umidade Relativa do Ar (\%) (UR) e Índice de Temperatura e Umidade (ITU), respectivamente. A média de Tbs foi de $29,4^{\circ} \mathrm{C}$, e a média do ITU, 76,2. Já a UR apresentou média de $45,6 \%$.

A amplitude dos dados fisiológicos obtidos em clima mediterrâneo foi de $68 \operatorname{mov} \min ^{1}$ e $2,1^{\circ} \mathrm{C}$ para a FR e TR, respectivamente, e a FR variou do mínimo de $36 \mathrm{mov} \mathrm{min}^{-1}$ ao máximo de $104 \mathrm{mov} \mathrm{min}^{-1}$, com média de $58,4 \mathrm{mov} \mathrm{min}^{-1}$. A TR variou do mínimo de $37,9^{\circ} \mathrm{C}$ ao máximo de $40,0^{\circ} \mathrm{C}$, sendo a média $38,5^{\circ} \mathrm{C}$. Já as variáveis climáticas Tbs e UR e o ITU apresentaram, respectivamente, uma amplitude de $18,7^{\circ} \mathrm{C}$ (mínimo de $19,5^{\circ} \mathrm{C}$ e máximo de $38,2^{\circ} \mathrm{C}$ ), 58,8\% (mínimo de $19,3 \%$ e máximo de 78,1\%) e 19,5 (mínimo de 66,3 e máximo de $85,8)$. A média da Tbs foi de $29,3^{\circ} \mathrm{C}$, a média da UR foi de $41,4 \%$ e a média do ITU foi de 75,4 .

Na segunda fase de análise dos dados, o conhecimento adquirido com a Mineração de Dados e transformado em uma base de regras foi utilizado para a modelagem do sistema inteligente, que foi baseado em lógica fuzzy. A figura 1 ilustra a base de regras no MatLab ${ }^{\circledR}$ com a situação de conforto térmico obtidacom os dados médios de temperatura retal e freqüência respiratória coletados na instalação estudada.

Cada regra foi combinada, e o resultado numérico foi defuzzificado, indicando uma possível ocorrência de estresse ou não. Portanto, existe uma

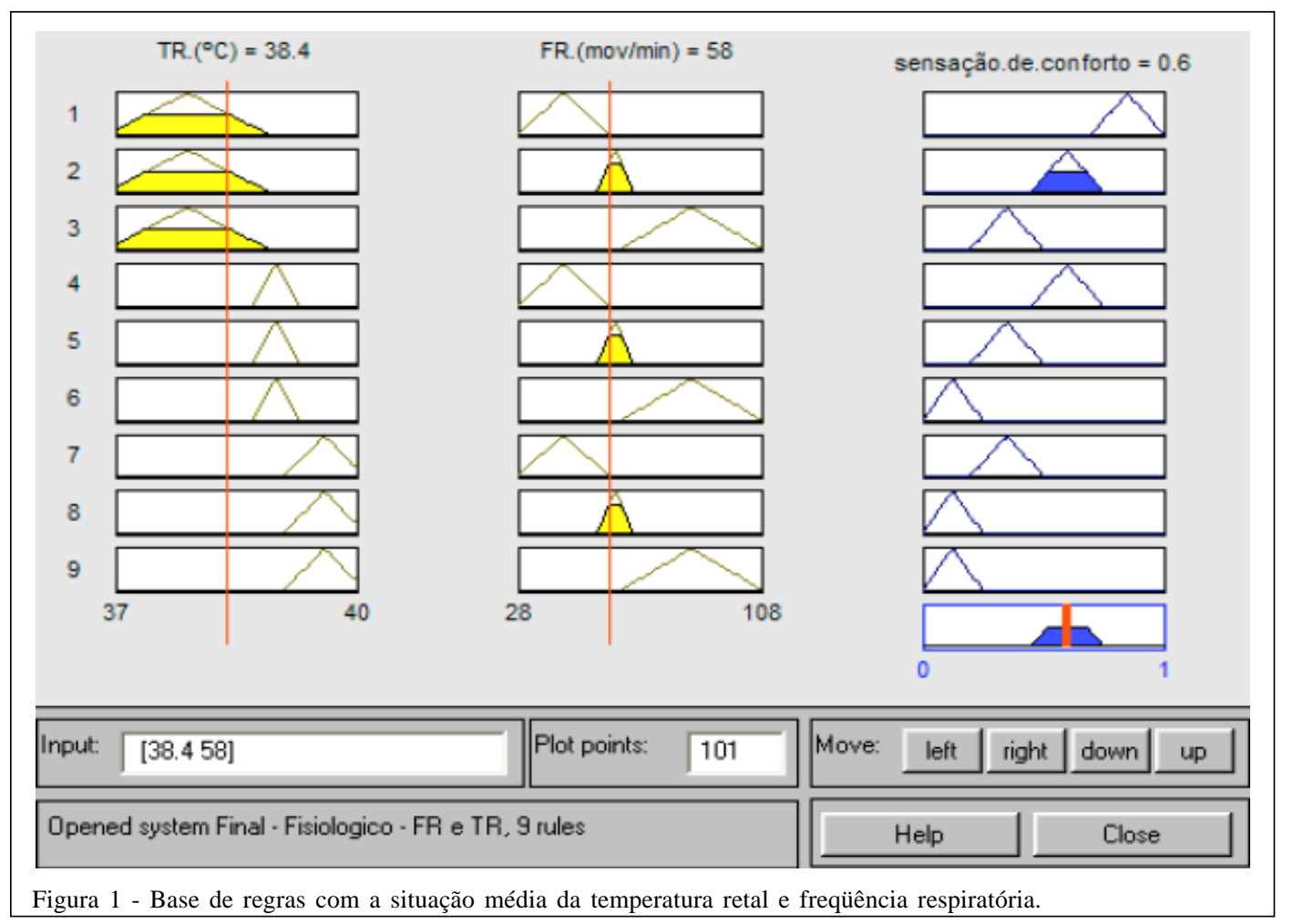

Ciência Rural, v.39, n.5, ago, 2009. 
tomada de decisão por meio dos valores obtidos e da visualização da base de regras, sabendo-se que dessa maneira é possível simular situações entre as variáveis de entrada (temperatura retal e freqüência respiratória) e ter uma resposta da variável de saída (conforto térmico animal), evidenciada na figura 1.

Como simulação, foram utilizados os valores médios das variáveis fisiológicas TR e FR obtidos durante o período experimental. No ambiente 1 , os valores médios da TR e da FR foram, respectivamente, $38,4^{\circ} \mathrm{C}$ e $58 \mathrm{mov} \mathrm{min}^{-1}$. A partir da base de regras estabelecidas, foi caracterizada uma condição de conforto classificada como boa, com o índice 0,6. A condição extrema máxima apresentou média de $38,6^{\circ} \mathrm{C}$ para a TR e 62,6mov minn-1 $^{-1}$ para a FR, sendo a condição de conforto também caracterizada como boa, com o índice 0,589 . Já para a condição extrema mínima, que apresentou os valores médios de $38,1^{\circ} \mathrm{C}$ e 49,4 mov $_{\text {minn }}^{-1}$ para a TR e para a FR, respectivamente, o índice encontrado foi de 0,85 , caracterizando uma condição desconforto muito boa.

Já no ambiente 2, os valores médios da TR e da FR foram, respectivamente, $38,5^{\circ} \mathrm{C}$ e $58,4 \mathrm{mov} \mathrm{min}^{-1}$. A partir da base de regras estabelecidas, foi caracterizada uma condição de conforto classificada como boa, com o índice 0,6. A condição extrema máxima apresentou média de $38,8^{\circ} \mathrm{C}$ para a TR e $65,9 \mathrm{mov} \mathrm{min}^{-1}$ para a FR, sendo a condição de conforto caracterizada como ruim, com o índice 0,218 . Já para a condição extrema mínima, que apresentou os valores médios de $38,3^{\circ} \mathrm{C}$ e $55,1 \mathrm{mov} \mathrm{min}^{-1}$ para a TR e para a FR, respectivamente, o índice encontrado foi de 0,728 , caracterizando uma boa condição de conforto térmico.

A partir dessa base de regras, obteve-se o gráfico tridimensional ilustrado na figura 2, com a variação não-linear de todas as situações possíveis de conforto térmico consideradas nesta pesquisa, por meio do qual é possível visualizar a sensação de conforto térmico como variável dependente e a temperatura retal e a freqüência respiratória como variáveis independentes.

De acordo com a figura 2, é possível observar, por meio da cor do gráfico, que quanto mais próximas da zona de conforto, mais as cores tendem ao amarelo e quanto mais as variáveis de entrada se afastam das faixas de conforto, mais azuis ficam no gráfico. Nesse caso, a sensação de conforto térmico é baixa.

O cruzamento dos dados fisiológicos temperatura retal e freqüência respiratória permitiu, por meio da classificação fuzzy, concluir que a observação de valores simultâneos de TR e a FR, menores ou iguais a $38,7^{\circ} \mathrm{C}$ e a $54 \mathrm{mov} \mathrm{min}^{-1}$, respectivamente, indicam um estado de conforto térmico dos animais muito bom. Nos casos em que a TR estiver na faixa de 38,7 a $39,2^{\circ} \mathrm{C}$, quando a FR for menor ou igual a $54 \mathrm{mov} \mathrm{min}^{-1}$ e em que a FR estiver na faixa de 54 a $66 \mathrm{mov} \mathrm{min}^{-1}$, quando a TR for menor ou igual a $38,7^{\circ} \mathrm{C}$, a sensação de conforto

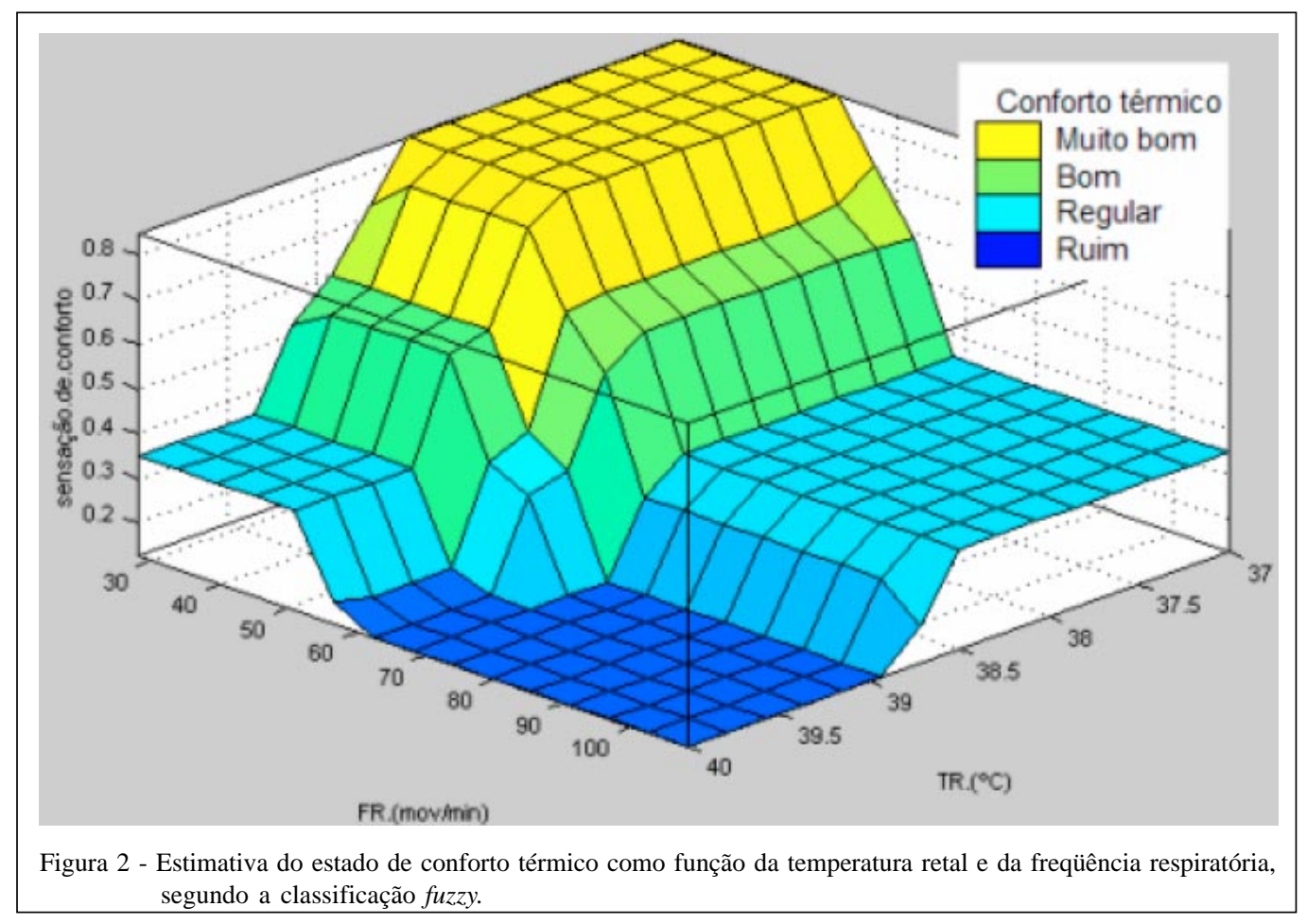

Ciência Rural, v.39, n.5, ago, 2009. 
poderá ser classificação como boa, concordando com HAHN et al. (1997), segundo o qual uma FR correspondente a $60 \mathrm{mov} \mathrm{min}^{-1}$ indicaria animais com ausência ou mínimo de estresse térmico. Os resultados também estão de acordo com PERISSINOTTO (2003), que não observou sinais de estresse térmico a valores médios de FR por volta de $55 \mathrm{mov}^{\mathrm{min}^{-1}}$ para vacas submetidas à climatização. Além disso, os resultados estão de acordo com MARTELLO (2006), que encontrou valores de FR durante os períodos mais quentes de primavera e verão de 50 e $58 \mathrm{mov} \mathrm{min}^{-1}$, respectivamente, sem constatar estresse térmico.

Já quando a FR for maior a $66 \mathrm{mov} \mathrm{min}^{-1}$ ao mesmo tempo em que a TR for maior a $38,7^{\circ} \mathrm{C}$, o conforto poderá ser classificado como ruim. Porém, caso a FR registre valores superiores a $66 \mathrm{mov} \mathrm{min}^{-1}$ e a TR registre valores menores a $38,7^{\circ} \mathrm{C}$, a sensação de conforto será regular. Do mesmo modo, uma TR com valores acima de $39,2^{\circ} \mathrm{C}$ poderá provocar um estado de conforto ruim, caso a FR apresente-se maior a $54 \mathrm{mov}$ minn $^{-1}$. Porém, valores de FR menores a $54 \mathrm{mov} \mathrm{min}^{-1}$ registrados ao mesmo tempo que valores de TR maiores a $39,2^{\circ} \mathrm{C}$ poderão estar representando uma sensação de conforto térmico regular. Um estado de conforto térmico regular também poderá ocorrer caso a FR apresente-se na faixa de 54 a 66 mov min $^{-1}$, simultaneamente a valores de TR na faixa de 38,7 a $39,2^{\circ} \mathrm{C}$.

As análises avaliadas no presente trabalho poderiam ser realizadas por outros meios que não a lógica fuzzy. Porém, a aplicação dessa ferramenta fornece um modelo de fácil interpretação. Além disso, os sistemas especialistas fuzzy aqui aplicados permitiram a integração do conhecimento científico de várias pesquisas e podem ser utilizados para auxiliar o entendimento do conforto térmico dos bovinos leiteiros. Segundo ALVES (2006), outra vantagem é a possibilidade de simples adaptação, por meio de mudanças de regras e funções de pertinência. Assim, vale destacar que novas regras podem ser facilmente integradas ao sistema, quando disponibilizadas por um especialista da área de estudo.

\section{CONCLUSÃO}

Concluiu-se que a aplicação da lógica fuzzy, na obtenção de parâmetros indicativos das condições de conforto térmico em bovinos leiteiros em lactação confinados, forneceu modelos de fácil interpretação, tornando-se uma ferramenta importante na compreensão das condições de conforto térmico dos animais ao associar a temperatura retal e a freqüência respiratória ao atendimento das necessidades de manutenção de conforto térmico. A utilização do software MATLAB ${ }^{\circledR}$ permitiu estabelecer alguns parâmetros ideais de conforto ambiental aos animais e condições de estudo. Sendo assim, os resultados mostraram que é possível o desenvolvimento de um software que determine, com elevado grau de precisão, a ocorrência do estresse térmico e o melhor momento para acionamento dos sistemas de climatização, de acordo com a resposta fisiológica dos animais e as condições climáticas de exposição, favorecendo diretamente as formas de manejo e reduzindo os custos de produção.

\section{REFERÊNCIAS}

AMENDOLA, M. et al. Análise matemática de condições de conforto térmico para avicultura usando a teoria dos conjuntos fuzzy. Biomatemática, v.14, n.1, p.87-92, 2004.

AMENDOLA, M. et al. Using fuzzy sets theory to analyze environmental conditions in order to improve the milk production. In: CONGRESSO NACIONAL DE MATEMÁTICA APLICADA E COMPUTACIONAL-CNMAC, 2006, Campinas, SP. Anais... Campinas: CNMAC-2006. V.1, p.1-1.

ALVES, S.P. Uso da zootecnia de precisão na avaliação do bem-estar bioclimático de aves poedeiras em diferentes sistemas de criação. 2006. 128f. Tese (Doutorado em Física do Ambiente Agrícola) - Curso de Pós-graduação em Física do Ambiente Agrícola. Escola Superior de Agricultura “Luiz de Queiroz”, Universidade de São Paulo.

AMENDOLA, M. et al. Using fuzzy sets theory to analyse environmental conditions in order to improve animal productivity. In: WORLD CONGRESS ON COMPUTATION IN AGRICUlTURE, 4., 2005a, Vila Real. Proceedings... Vila Real: UEVORA, 2005a. V.1, p. 1-8.

AMENDOLA, M. et al. Manual do uso da teoria dos conjuntos Fuzzy no MATLAB 6.5. Campinas: UNICAMP, FEAGRI \& IMECC, 2005b. 46p. Capturado em 4 mai. 2007. Online. Disponível em: <http://www.ime.unicamp.br/ laeciocb/ manual_fuzzy_matlab.pdf $>$.

BRUNASSI, L. A. et al. Teste de um sistema fuzzy de identificação de estro em uma fazenda comercial de vacas leiteiras. In: SIMPÓSIO DE CONSTRUÇÕES RURAIS E AMBIENTES PROTEGIDOS, SIMCRA 2006, Campinas, SP. Anais... Campinas: FEAGRI/UNICAMP, 2006. 1 CD.

CHAPMAN, P. et al. CRISP-DM 1.0. Step-by-step data mining guide. 2000. 78p. Capturado em 10 Abr. 2007. Online. Disponível em: <http://www.crisp-dm.org/CRISPWP-0800.pdf>.

CHIARINI, A.M. et al. Uso da lógica fuzzy para o desenvolvimento de um modelo padrão de avaliação do ambiente de maternidade de suínos. In: SIMPÓSIO DE CONSTRUÇÕES RURAIS E AMBIENTES PROTEGIDOS, SIMCRA 2006, Campinas, SP. Anais... Campinas: FEAGRI/UNICAMP, 2006. 1 CD.

GATES, R.S. et al. Fuzzy control simulation of plant and animal environments. In: ASAE ANNUAL INTERNATIONAL MEETING, 1999, Toronto, Canada. 24p. 
Capturado em 10 Nov. 2006. Online. Disponível em: capeta.<http://www.bae.uky.edu/gates/freebies/ASAE99/ 993136.pdf $>$.

HAHN, G.L. et al. Cattle respiration rate as a function of ambient temperature. Transactions of American Society of Agricultural Engineering, v.40, p.97-121, 1997.

MANDANI, E.H. Advances in the linguistic syntesis of fuzzy controllers. International Journal of Man-Machine Studies, v.8, n.6, p.669-678, 1976.

MARTELLO, L.S. Interação animal-ambiente: efeito do ambiente climático sobre as respostas fisiológicas e produtivas de vacas Holandesas em free-stall. 2006. 113f. Tese (Doutorado em Qualidade e Produtividade Animal) - Curso de Pós-graduação em Zootecnia.,Faculdade de Zootecnia e Engenharia de Alimentos, Universidade de São Paulo.

MOTA, L.S. Adaptação e interação genótipo-ambiente em vacas leiteiras. 1997. 69f. Tese (Doutorado em Ciências) - Curso de Pós-graduação em Biologia Comparada, Faculdade de Medicina de Ribeirão Preto, Universidade de São Paulo.

OLIVEIRA, H.L. et al. Estimated thermal comfort condition for layers according to fuzzy theory. Engenharia Agrícola, v.25, n.2, p.300-307, 2005.

OWADA, A.N.; NÄÄS, I.A. Utilização da lógica fuzzy para avaliação do bem-estar de frangos de corte. In: SIMPÓSIO DE CONSTRUÇÕES RURAIS E AMBIENTES PROTEGIDOS,
SIMCRA 2006, Campinas. SP. Anais... Campinas: FEAGRI/ UNICAMP, 2006. 1 CD.

PANDORFI, H. et al. Uso da tecnologia da informação para análise de sinais e padrões com ênfase no conforto e bem-estar de matrizes suínas gestantes. In: REUNIÃO ANUAL DA SOCIEDADE BRASILEIRA DE ZOOTECNIA, 43., 2006, João Pessoa, PB. Anais... João Pessoa: SBZ, 2006. 1 CD.

PERISSINOTTO, M. Avaliação da eficiência produtiva e energética de sistemas de climatização em galpões tipo freestall para confinamento de gado leiteiro. 2003. 140f. Dissertação (Mestrado em Física do Ambiente Agrícola) - Curso de Pós-graduação em Física do Ambiente Agrícola. Escola Superior de Agricultura "Luiz de Queiroz”, Universidade de São Paulo.

SANTOS, R.C.; NÄÄS, I.A. Utilização da lógica fuzzy para a simulação do estro de bovino leiteiro exposto a diferentes valores de temperatura e UR. In: SIMPÓSIO DE CONSTRUÇÕES RURAIS E AMBIENTES PROTEGIDOS, 2006, Campinas, SP. Anais... Campinas: FEAGRI/UNICAMP, 2006. 1 CD.

THOM, E. C. The discomfort index. Weatherwise, v.12, p.57-59, 1959.

WATANABE, B.M. et al. Análise das condições de conforto de frangos de corte utilizando a teoria dos conjuntos fuzzy. In: WORKSHOP DA PÓS-GRADUAÇÃO DA FEAGRI/UNICAMP, 5., 2005, Campinas, SP. Anais... Campinas, FEAGRI/ UNICAMP, 2005. 1 CD. 